\title{
Sarcoporosis Is a Part of Aging
}

\section{Petr Broulík}

$3^{\text {rd }}$ Department of Medicine - Department of Endocrinology and Metabolism, First Faculty of Medicine, Charles University and General University Hospital in Prague, Prague, Czech Republic

Received March 8, 2019; Accepted September 4, 2019.

Key words: Aging - Sarcopenia - Osteoporosis - Frailty

Abstract: Ageing is associated with the accumulation of damage to all the macromolecules within and outside cells leading to progressively more cellular and tissue defects and resulting in age-related frailty, disability and disease. As a result of the aging process the bone deteriorates in composition, structure and function. Age-related musculoskeletal losses are a major public health burden because they can cause physical disability and increased mortality. We tried to find out on a small set of old women, without risk factors for osteoporosis, what caused them the loss of bone minerals. All 492 women had just only one risk factor - the old age. Laboratory findings have shown a decreased serum $C$ telopeptide and low serum alkaline phosphatase circulating markers, used to quantify bone resorption and formation, and very low level of vitamin D. Very low level of vitamin D that disrupted calcium absorption through the intestine, and decreased calcemia increased parathyroid hormone levels with resulting bone effect. The manifestation of physiological aging is worsening eyesight, peripheral neuropathy, depression, worsening of physical condition, skin aging, sarcopenia and bone mineral loss. Senile osteoporosis, which is not caused by known risk factors for osteoporosis, does not appear to be a separate disease, but is part of the physiological process of aging. Treatment of senile osteoporosis should be focused on the control of secondary hyperparathyroidism by administration of vitamin $\mathrm{D}$ and calcium. The risk of fractures in the advanced age is determined by a large number of factors ranging from hazards in the home environment to frailty and poor balance.

Mailing Address: Prof. Petr Broulík, MD., DSc., $3^{\text {rd }}$ Department of Medicine Department of Endocrinology and Metabolism, First Faculty of Medicine, Charles University and General University Hospital in Prague, U Nemocnice 1, 12808 Prague 2, Czech Republic; Phones: +420 224966 686, +420 224962 954; e-mail: pbrou@lf1.cuni.cz 


\section{Introduction}

The very old age is difficult to define. It is the last phase of life, in which the involution, the sum of the involutional changes (extinction, "drop", atrophic), with deterioration of the fitness, the resistance and the adaptability of the organism is more pronounced. We are talking about frailty syndrome. Frailty was proposed by Fried and colleagues (2001) using data from the cardiovascular health study and is based on five characteristics: unintentional weight loss, muscle weakness, reduced energy and endurance, slowness of gait and low physical activity.

The body changes with aging because changes occur in individual cells and in whole organs. These changes result in changes in function and in structure. When the number of cells becomes too low, an organ cannot function normally. Thus, most organs during aging start to function sub-optimally. The manifestations of physiological aging are deteriorating vision, peripheral neuropathy, depression, deconditioning, skin aging, sarcopenia, osteoporosis and movement disorders. Age-related musculoskeletal losses are a major public health burden because they can cause physical disability and increased mortality.

Bones become less dense partly because they contain less calcium (which gives bones strength). The amount of calcium decreases because the body absorbs less calcium from foods. Also, levels of vitamin $\mathrm{D}$, which helps the body use calcium, decrease. Certain bones are weakened more than others. Those most affected include the end of the thighbone (femur) at the hip, the ends of the arm bones (radius and ulna) at the wrist, and the bones of the spine (vertebrae).

Osteoporosis (OP) is predominantly a condition of the elderly although the age profile varies for different fractures. Senile osteoporosis is a type of osteoporosis, a disease of bone leading to an increased risk of fracture due to reduced bone density in aging people. There is calcium deficiency which leads to deterioration of the bone structure, involves a thinning of both the trabecular (spongy) and cortical (hard) bone.

The study has been undertaken to find out if the old age without other risk factors for osteoporosis can be the only cause for involutional osteoporosis. The primary efficacy endpoint was to find out if there are women with low bone mineral content who are just only old.

\section{Methods}

We studied 492 women who were recruited from 3,000 patients treated at the $3^{\text {rd }}$ Department of Medicine - Department of Endocrinology and Metabolism, First Faculty of Medicine, Charles University and General University Hospital in Prague during the years 2016 and 2017. Women who were 75 years of age or older were considered to be eligible if they had received first time a diagnosis of osteoporosis and did not have known risk factors for osteoporosis and who had been visited on our outpatient ward for first time. 
Women were excluded if they had hypercalcemia, hypocalcemia, recent use of drugs affecting bone metabolism or have hysterectomy and adnexotomy or if they had taken estrogen, or glucocorticoids within the preceding 2 years or bisphosphonates at any time.

At the first visit to the outpatient ward medical history was recorded and all patients underwent physical examination. A study questionnaire was used for recording the relevant demographic and clinical data (age, weight, height, abdominal girth, smoking habit, medications and concomitant disease).

All patients provided written informed consent and the protocol was approved by the appropriate ethical review boards.

The research group consists of 492 women aged $75-92$ years with an average age of $80 \pm 7$ years, with a T-score of more than -2.5 SD (standard deviation) at the lumbar spine or total hip or femoral neck were eligible for participation. Clinical risk factors used for the assessment of fracture probability were: previous fragility fracture, glucocorticoid treatment, current smoking, untreated hypogonadism, inflammatory bowel disease, prolonged immobility, thyroid disorders and HIV infection (Kanis et al., 2019).

Standing height and weight measurements were completed with participants wearing lightweight clothing and no shoes. Body mass index (BMI) was calculated. Standard laboratory tests were used to determine levels of calcium, phosphate alkaline phosphatase, gamma glutamyl transferase and cholesterol.

Laboratory parameters used to evaluate changes in bone turnover were serum $C$-terminal telopeptide of collagen I ( $\beta C T X)$ to assess the rate of bone resorption and serum alkaline phosphatase with GGTP (gamma glutamyl transpeptidase) to assess the rate of bone formation. Assays were performed at the central laboratory.

Bone mineral density (BMD) was measured at the lumbar spine (L1-L4), total femur (TF), femoral neck (FN), in all participants by using a dual energy $X$-ray absorptiometry (DXA) densitometer (Discovery A, Hologic, Inc., MA, USA, Software 6 version: Apex 3.5.1) or DEXA Lunar Prodigy. Patients and control groups were measured on the same densitometer. BMD was expressed as $\mathrm{g} / \mathrm{cm}^{2}$ of hydroxyapatite and as T-scores (standard deviation from the mean of a healthy population of the same age).

Spine radiographs were evaluated at a central site. Screening spine radiographs were evaluated for the presence or absence of a vertebral fracture using the semiquantitative scale (Genant et al., 1993). Atraumatic fractures were defined as fracture following insignificant antecedent trauma such as bending, twisting, sneezing or coughing.

\section{Results}

All results are given in Table 1. The present study included a total of 492 women with age $80 \pm 7$ years. The women had low normal calcium, high normal parathyroid hormone, very low concentration of $25(\mathrm{OH}) \mathrm{D}$ and normal phosphate. 


\section{Table 1 - Clinical and biochemical data from 492 women with osteoporosis (means \pm SD)}

\begin{tabular}{lc}
\hline & Number -492 \\
\hline Sex (female) & 492 \\
Age $($ years $)$ & $80 \pm 7$ \\
BMI $\left(\mathrm{kg} / \mathrm{m}^{2}\right)$ & $28.8 \pm 3.6$ \\
S. calcium $(\mathrm{mmol} / \mathrm{I})(2.0-2.7)$ & $2.34 \pm 0.2$ \\
S. phosphate $(\mathrm{mmol} / \mathrm{I})(0.65-1.61)$ & $1.16 \pm 0.3$ \\
S. PTH $(\mathrm{pmol} / \mathrm{I})(1.58-6.03)$ & $6.2 \pm 2.8$ \\
Uric acid $(\mathrm{mmol} / \mathrm{I})(220-420)$ & $368 \pm 45$ \\
S. creatinine $(\mu \mathrm{mol} / \mathrm{I})(44-110)$ & $74 \pm 5$ \\
S. total cholesterol $(\mathrm{mmol} / \mathrm{I})(2.9-5.2)$ & $6.23 \pm 0.6$ \\
Calcidiol $(\mathrm{ng} / \mathrm{ml})(30-80)$ & $10.6 \pm 5.2$ \\
CTx $($ ug/l) $(0-1008)$ & $525 \pm 170$ \\
Polymorbidity & $468(96 \%)$ \\
Degenerative changes in axial skeleton and supporting joins & $482(98 \%)$ \\
ALP $(\mu$ kat/l) $(0.65-2.20)$ & $1.39 \pm 0.14$ \\
GGTP $(\mu$ kat/l) $(0.14-0.84)$ & $0.38 \pm 0.02$ \\
Back pain & $482(98 \%)$ \\
Fracture presented on X-ray & $45(9 \%)$ \\
\hline
\end{tabular}

SD - standard deviation; BMI - body mass index; S. - serum; PTH - parathyroid hormone; CTx - C-terminal telopeptide; ALP - alkaline phosphatase; GGTP - gamma glutamyl transpeptidase

Circulating biochemical marker of bone formation (gamma glutamyl transferase and alkaline phosphatase) was not significantly higher. The marker of bone resorption serum CTx was normal not suggesting increase in bone remodelling.

The women had normal cholesterol and had overweight. 45 women (9\%) had one radiologically defined fracture.

Bone mineral density in femoral neck and lumbar spine measured by T-score has been in the range of osteoporosis (T-score $<-2.5 \mathrm{SD}$ ).

\section{Discussion}

As a result of the aging process the bone deteriorates in composition, structure and function (Demontiero et al., 2012). The mineral composition of bone changes with advancing age. Bone matrix, the framework of skeletal cells, becomes weaker and thinner. With advancing age, a negative balance in bone remodelling results in decreased bone mass and alterations in the bone structure. The amount of bone resorbed by the osteoclasts is not fully restored with bone deposited by the osteoblasts and this imbalance leads to bone loss. Loss of bone mass and strength in humans with advancing age, is associated with an increase in the prevalence of apoptotic osteoblasts and osteocytes and a corresponding decrease in osteoblast number and decreased bone formation rate (Almeida, 2012). Several studies have reported considerable variations in the shape of osteocytes and their lacunae 
with aging. The osteocytes are considered to be the cells responsible for sensing mechanical signals on the bones and consequently orchestrating the activity of osteoblasts and osteoclasts (Hemmatian et al., 2017). Senile osteoporosis, also referred to as degenerative osteoporosis, occurs as a result of aging on the bones. Most of bone mass in human body is the cortical bone. Typical for very old people is the excessive cortical bone thinning and risk of nonvertebral fractures. Nonvertebral fractures form $75 \%$ of all fractures after 60 years of age (Bliuc et al., 2014). Bone is a tissue with continual active metabolic turnover associated with its remodelling (Riggs et al., 2004). Increased bone resorption leads to the initial fall in bone mineral density. With increasing age there is also a significant reduction in bone formation. This is mostly due to a shift from osteoblastogenesis to predominant adipogenesis in the bone marrow. There is predominant differentiation of mesenchymal stem cells (MSCs) into adipocytes at the expense of osteoblasts (Rosen et al., 2009). The lipotoxicity of marrow adipocytes on bone comes from the observation of PPAR $\gamma$ induction by thiazolidenediones (Broulík et al., 2011). With aging there is a lower levels of osteoblast differentiation (Lecka-Czernik, 2006).

As a person ages, especially after crossing the age of 70 , there is impairment in the function of the kidneys with decreased absorption and ability to synthetize vitamin D. The decreased concentration of vitamin D hinders the amount of calcium which can be absorbed. Decreased calcium levels trigger the parathyroid hormone to send signals to the body to reabsorb the bone in order to compensate for the deficiency of calcium in the body. All this results in gradual eroding of the spongy and hard bone structure with a resultant increased risk of bone fractures (Demontiero et al., 2012). New bone formation slows down with increasing age, and the rate by which old bone cells are reabsorbed increases. Age-related factors in etiopathogenesis of senile osteoporosis include low intake of vitamin $D$ and reduced capacity to synthesize vitamin $D$ in skin of elderly individuals. At the age of 70 , the activity of enzymatic apparatus of the skin synthetizing vitamin $D$ is up to 10-times lower than in young individuals. Vitamin $D$ is not common in food. With increasing age hydroxylation of vitamin $D$ slows down and resistance of target tissue to the active vitamin D metabolite calcitriol increases. Lower levels of active vitamin D metabolite lead to reduced intestinal absorption of calcium and hypocalcemia, stimulating production and release of parathyroid hormone (PTH) by parathyroid glands; the patients' levels of immunoreactive PTH (senile secondary hyperparathyroidism) are increasing. There occurs imbalance in bone remodelling in favour of bone resorption, with marked multiplication of resorption cavities and their inadequate filling with osteoblasts.

Lack of vitamin D and its active metabolites also results in reduced muscle strength, up to 4-times in the quadriceps, and limited neuromuscular coordination. Muscle weakness contribute to fatigue, weakness and reduced activity tolerance. Sarcopenia recently has been defined as reduced skeletal muscle mass associated with low muscle strength poor physical performance or both (Cruz-Jentoft et al., 
2019). The amount of muscle tissue and muscle strength tend to decrease beginning around age 30 and continuing throughout life (Kemp et al., 2018). Sarcopenia and osteoporosis are both processes associated with aging caused by a decrease in mass and quality, with very similar etiological moments and very similar pathogenesis. Analyzes of geriatric patients show that the vast majority of them suffer from both sarcopenia and osteoporosis. Some of people speak of sarcoporosis (Jenšovský, 2012).

Joint problems ranging from mild stiffness to debilitating arthritis have been very common in our women. Additionally, the use of medications and the presence of health problems increases as people grow older, and these things may interfere with how the body uses nutrients needed for bone health. Some medications and health conditions prevent absorption of minerals. Collagen formation may become impaired.

In the aging process, bone is also adversely affected by decrease in production of bone anabolic peptides, such as growth hormone, insulin and insulin growth factor I, and adrenal steroids, such as dehydroepiandrosterone and androstenedione.

An important factor with a negative impact on bone mass is immobilization, even if short-term, when due to insufficient stimulation of bone mechanoreceptors, bone resorption prevails over bone formation. Elderly persons have often their joint apparatus damaged by degenerative diseases limiting their mobility, ability to walk and protracting periods of immobility, which are significant risk factors for development of osteoporosis.

Of a great interest is the relation between blood circulation and bone metabolism. Ischemia caused by ligature of the femoral artery in rabbits resulted in cortical thinning and reduction of the mechanical resistance of the bone. Laroche et al. (1995) found atherosclerotic changes in interosseous arteries that were similar to those in coronary arteries.

Bones receive about $5 \%$ of cardiac output. A limiting factor for centrifugal resorption is most probably the demand of most remote cells for adequate supply of oxygen and amino acids, and the possibilities of removing waste products. Of great importance for bone cells and their metabolic activity is the regional blood flow. Our group of women with compression fractures of vertebral bodies exhibited a higher incidence of ischemic heart disease or ischemic disease of lower limbs (Broulik et al., 1982).

BMD decreases with the patient's increasing age, $50 \%$ of women in 70-79-year age group have T-score of bone density in the femoral neck less than -2.0 SD and at the age of 80 years and more their number increases up to $70 \%$. In patients over 75 years, Z-score rather than T-score should be taken into account. However, experienced osteologists do not focus merely on densitometry that may be associated with a number of errors (degenerative changes in the spine) especially in the region of the L-spine, but assess all indicators of the disease, including the patient's habitus (Schousboe et al., 2013). 
In elderly patients, the predictive value of densitometry assessment of the proximal femur is much higher. BMD measuring of the proximal femur and femoral neck has shown that a higher incidence of fractures in these locations is associated with a lower BMD value. Although there are not many longitudinal studies measuring BMD in the elderly, it seems that in some patients, loss of the cortical, and perhaps also trabecular, bone stops after the age of 70 .

Elderly patients with senile osteoporosis should be diagnosed on a case-by-case basis, as in advanced age a number of severe degenerative changes can be found in the spine concomitantly with osteoporosis that may play a significant role in explaining the patients' complaints.

The treatment of involutional osteoporosis is currently much more effective in prevention of bone loss than in its restoration. Treatment of elderly patients is less effective than in the young individuals. Data on treatment of osteoporosis reported in the literature relate almost exclusively to the population younger than 75 years. It should be noted that the speed of loss of the cortical and trabecular bone changes with increasing age and its response to treatment varies. Trabecular bone is metabolically more active than cortical bone and therefore its response to treatment is different (Boonen et al., 2008).

Treatment of senile osteoporosis should be focused on the control of senile secondary hyperparathyroidism by administration of vitamin $D$ and calcium. Patients receive vitamin D3 either in drops (14-20 gtto once a week), or injections of cholecalciferol (vitamin D2) 300,000 IU i.m. once a month that have proved to be effective as they are more regular. However, vitamin D3 is four-time more powerful. The given protocol of administration of vitamin $\mathrm{D}$ prevents overdosing because it has been demonstrated that the level of active vitamin D 25(OH)D3 is extremely low in the elderly (Rizzoli et al., 2013). The optimum dose of vitamin D3 in the elderly is $800 \mathrm{IU}$ per day. It is recommended to administer vitamin D to seniors as the prevention (Hin et al., 2017). In addition, it is necessary to ensure the daily intake of calcium, i.e. at least 500-1,000 mg of elemental calcium per day. Some polymorbid patients may have problems with calcium intake (intolerance, constipation, lack of appetite). This condition may be addressed by food high in calcium, milk cheese and white yoghurts. It is also possible to combine calcium supplements with foods rich in calcium. Calcium carbonicum requires an acidic environment, and therefore is administered during meals (Caution is necessary in case of administration of blockers of H2-receptor or proton pump!) (Pfeifer et al., 2009).

Certain cases of severe osteoporosis with pain syndrome were successfully treated with intravenous injections at the dose of 5 vials of $10 \%$ calcium gluconicum with one vial of quajacurane or tramal (personal experience). The cycle includes up to 10 such infusions. Combined administration of 1,200 mg of calcium per day with a physiological dose of vitamin D3 significantly decreases the incidence of non-vertebral fractures, including proximal femur fractures, in individuals older than 75 years (Bischoff-Ferrari et al., 2009). 
It is also possible to use hydroxyapatitum osseum which contains both inorganic (hydroxyapatite) and organic (ossein) components. A similar preparation is made from eggshells.

The above mentioned preparations together with specialized rehabilitation, exercises, massage and iontophoresis are essential tools in treatment of senile osteoporosis.

Of no less importance is a proper diet with adequate amount of proteins. People with positive protein balance heal much better following surgery, such as total hip replacement. A high intake of salt increases calciuria values, similarly as excessive intake of proteins. A diet rich in salt and animal proteins may increase the patient's calcium requirement (Mangano et al., 2017).

It is important for the patients to follow the rehabilitation plan, including exercises to strengthen lower limb muscles and to improve gait and postural stability. Elderly patients should be adequately informed about all environmental safety hazards in their homes, particularly in the bathroom, leading to potential falls (Scherrington et al., 2008).

A favourable effect of rehabilitation and bone loading exercises is explained by irritation of bone cells responsible for bone formation, namely by electrical current induced by activation of bone crystals. Another favourable effect of exercises is remodelling of bone trabeculae in the direction of the highest loading. Elderly patients experience especially in the region of an osteoporotic bone affected by microfractures, muscle spasms that cause pain syndrome.

It has to be taken into account that individuals aged 80 and more are highly susceptible to fractures resulting from falls, even if they receive adequate treatment. The risk of fracture of the arm, forearm, femoral neck or vertebral body increases between the age of 45 and 85 years 8 -times in women and 5 -times in men. About $30 \%$ of persons older than 65 years and $50 \%$ of persons older than 80 years fall at least once a year. A total of $90 \%$ of all proximal femur fractures are caused by fall. It is necessary to control factors leading to falls, i.e. gait and stability disorders (arthropathy, peripheral neuropathy, impairment of the ocular or vestibular system), postural hypotension (treatment of hypertension by diuretics), arrhythmia and the use of hypnotics, sedatives, anxiolytics and antidepressants (Kannus et al., 2004).

Hormone therapy should not be used in senile osteoporosis, as recommended by the recent Women Health Initiative (WHI) study. In elderly women there occurs pain in atrophic mammary gland, proliferation of atrophic endometrium with bleeding; the risk of ischemic heart disease, phlebothrombosis, hypertension and stroke are increasing.

Reasonable doses of anabolics have proved to be beneficial in senile osteoporosis. They considerably strengthen the muscle corset of the axial skeleton and their androgenic component has a favourable effect on osteoblasts. A certain role is played also by their euphorizing effect. Currently the market offers second- 
generation bisphosphonates - alendronate sodium, and third-generation bisphosphonates - ibandronate and zoledronate. These medications have a powerful antiresorptive effect targeted directly at osteoclasts, resulting in marked decrease of bone resorption, however our old patients suffering from senile osteoporosis do not have increased markers of osteoresorption. Data from Axelsson et al. (2017) show that in old patients with prior fracture alendronate reduce the risk of hip fracture. The study has limitation there is lack of data regarding bone densitometry and fracture trauma type. Data from Vandenbroucke et al. (2017) are overview of all possibilities how to treat osteoporosis. Paper support our idea that nonpharmacological interventions such as fall prevention play an essential role in the management of osteoporosis. These medications require a good knowledge of the patients in terms of their ability to comply with the prescribed use of medications and of their other comorbidities that should not be so severe to decrease the therapeutic efficacy of bisphosphonates. The approach must be strictly individualized, based on the knowledge of the patient's medical history, social status, possible polymorbidities, hearing and sight abilities and finally the ability to adhere to therapy (Black et al., 2012).

Pain caused by both osteoporosis and degenerative changes of the spine or weight bearing joints should be managed by a reasonable analgesic therapy that should relieve pain without affecting the patients' mobility and sense of orientation.

From our observation of 492 women with senile osteoporosis, which is not caused by known risk factors for osteoporosis, does not appear to be a separate disease, but is part of the physiological process of aging. Treatment of senile osteoporosis should be focused on the control of senile secondary hyperparathyroidism by administration of vitamin $\mathrm{D}$ and calcium.

\section{References}

Almeida, M. (2012) Aging mechanisms in bone. Bonekey Rep. 1, 102.

Axelsson, K. F., Wallander, M., Johansson, H., Lundh, D., Lorentzon, M. (2017) Hip fracture risk and safety with alendronate treatment in the oldest-old. J. Intern. Med. 282, 546-559.

Bischoff-Ferrari, H. A., Kiel, D. P., Dawson-Hughes, B., Orav, J. E., Li, R., Spiegelman, D., Dietrich, T., Willett, W. C. (2009) Dietary calcium and serum 25-hydroxyvitamin D status in relation to BMD among US adults. J. Bone Miner. Res. 24, 935-942.

Black, D. M., Bauer, D. C., Schwartz, A. V., Cummings, S. R., Rosen, C. J. (2012) Continuing bisphosphonate treatment for osteoporosis - For whom and for how long? N. Engl. J. Med. 366, 2051-2053.

Bliuc, D., Nguyen, T. V., Eisman, J. A., Center, J. R. (2014) The impact of nonhip nonvertebral fractures in elderly women and men. J. Clin. Endocrinol. Metab. 99, 415-423.

Boonen, S., Dejaeger, E., Vanderschueren, D., Venken, K., Bogaerts, A., Verschueren, S., Milisen, K. (2008) Osteoporosis and osteoporotic fracture occurrence and prevention in the elderly: a geriatric perspective. Best Pract. Res. Clin. Endocrinol. Metab. 22, 765-785.

Broulik, P., Kragstrup, J., Mosekilde, L., Melsen, F. (1982) Osteon cross-sectional size in the iliac crest: Variation in normals and patients with osteoporosis, hyperparathyroidism, acromegaly, hypothyroidism and treated epilepsia. Acta Pathol. Microbiol. Immunol. Scand. A 90, 339-344. 
Broulík, P., Sefc, L., Haluzík, M. (2011) Effect of PPAR- $\gamma$ agonist rosiglitazone on bone mineral density and serum adipokines in C57BL/6 male mice. Folia Biol. (Praha) 57, 133-138.

Cruz-Jentoft, A. J., Bahat, G., Bauer, J., Boirie, Y., Bruyère, O., Cederholm, T., Cooper, C., Landi, F., Rolland, Y., Sayer, A. A., Schneider, S. M., Sieber, C. C., Topinkova, E., Vandewoude, M., Visser, M., Zamboni, M. (2019) Sarcopenia: revised European consensus on definition and diagnosis. Age Ageing 48 , $16-31$.

Demontiero, O., Vidal, C., Duque, G. (2012) Aging and bone loss: new insights for the clinician. Ther. Adv. Musculoskelet. Dis. 4, 61-76.

Fried, L. P., Tangen, C. M., Walston, J., Newman, A. B., Hirsch, C., Gottdiener, J., Seeman, T., Tracy, R., Kop, W. J., Burke, G., McBurnie, M. A.; Cardiovascular Health Study Collaborative Research Group (2001) Frailty in older adults: evidence for a phenotype. J. Gerontol. A Biol. Sci. Med. Sci. 56, M146-M156.

Genant, H. K., Wu, C. Y., van Kuijk, C., Nevitt, M. C. (1993) Vertebral fracture assessment using a semiquantitative technique. J. Bone Miner. Res. 8, 1137-1148.

Hemmatian, H. I., Bakker, A. D., Klein, N., van Lenthe, G. H. (2017) Aging, osteocytes, and mechanotransduction. Curr. Osteoporos. Rep. 15, 401-411.

Hin, H., Tomson, J., Newman, C., Kurien, R., Lay, M., Cox, J., Sayer, J., Hill, M., Emberson, J., Armitage, J., Clarke, R. (2017) Optimum dose of vitamin D for disease prevention in older people. BEST-D trial of vitamin D in primary care. Osteoporos. Int. 28, 841-851.

Jenšovský, J. (2012) Bone care in older age. Interní Med. 14, 199-202. (in Czech)

Kanis, J. A., Cooper, C., Rizzoli, R., Reginster, J. Y.; Scientific Advisory Board of the European Society for Clinical and Economic Aspects of Osteoporosis and Osteoarthritis (ESCEO) and the Committees of Scientific Advisors and National Societies of the International Osteoporosis Foundation (IOF) (2019) Executive summary of the European guidance for the diagnosis and management of osteoporosis in postmenopausal women. Calcif. Tissue Int. 104, 235-238.

Kannus, P., Sievanen, H., Palvanen, M., Järvinen, T., Parkkari, J. (2004) Prevention of falls and consequent injuries in elderly people. Lancet 366, 1885-1893.

Kemp, G. J., Birrell, F., Clegg, P. D., Cuthbertson, D. J., De Vito, G., van Dieën, J. H., Del Din, S., Eastell, R., Garnero, P., Goljanek-Whysall, K., Hackl, M., Hodgson, R., Jackson, M. J., Lord, S., Mazzà, C., McArdle, A., McCloskey, E. V., Narici, M., Peffers, M. J., Schiaffino, S., Mathers, J. C. (2018) Developing a toolkit for the assessment and monitoring of musculoskeletal ageing. Age Ageing 47, iv1-iv19 (Suppl. 4).

Laroche, M., Ludor, I., Thiechart, M., Arlet, J., Pieraggi, M., Chiron, P., Moulinier, L., Cantagrel, A., Puget, J., Utheza, G. (1995) Study of the intraosseous vessels of the femoral head in patients with fractures of the femoral neck or osteoarthritis of the hip. Osteoporos. Int. 5, 213-217.

Lecka-Czernik, B. (2006) PPARs and bone metabolism. PPAR Res. 2006, 18089.

Mangano, K. M., Sahni, S., Kiel, D. P., Tucker, K. L., Dufour, A. B., Hannan, M. T. (2017) Dietary protein is associated with musculoskeletal health independently of dietary pattern: the Framingham Third Generation Study. Am. J. Clin. Nutr. 105, 714-722.

Pfeifer, M., Begerow, B., Minne, H., Tucker, K. L., Dufour, A. B., Hannan, M. T. (2009) Effects of a long-term vitamin $\mathrm{D}$ and calcium supplementation on falls and parameters of muscle function in community-dwelling older individuals. Osteoporos. Int. 20, 315-322.

Riggs, B. L., Melton, L. J. III., Robb, R. A., Camp, J. J., Atkinson, E. J., Peterson, J., Roulean, P. A., McCollough, C. H., Bouxsein, M. L., Khosla, S. (2004) A population-based study of age and sex differences in bone volumetric density, size, geometry and structure at different skeletal sites. J. Bone Miner. Res. 19, 1945-1954.

Rizzoli, R., Boonen, S., Brandi, M. L., Bruyère, O., Cooper, C., Kanis, J. A., Kaufman, J. M., Ringe, J. D., Weryha, G., Reginster, J. Y. (2013) Vitamin D supplementation in elderly or postmenopausal women: 
94) Prague Medical Report / Vol. 120 (2019) No. 2-3, p. 84-94

a 2013 update of the 2008 recommendations from the European Society for Clinical and Economic Aspects of Osteoporosis and Osteoarthritis (ESCEO). Curr. Med. Res. Opin. 29, 305-313.

Rosen, C., Ackert-Bicknell, C., Rodriguez, J., Pino, A. (2009) Marrow fat and the bone microenvironment: Developmental, functional, and pathological implications. Crit. Rev. Eukaryot. Gene Expr. 19, 109-124.

Schousboe, J. T., Shepherd, J. A., Bilezikian, J. P., Baim, S. (2013) Executive summary of the 2013 International Society for Clinical Densitometry Position Development Conference on bone densitometry. J. Clin.

Densitom. 16, 455-466.

Sherrington, C., Whitney, J. C., Lord, S. R., Herbert, R. D., Cumming, R. G., Close, J. C. (2008) Effective exercise for prevention of falls: a systemic review and meta-analysis. J. Am. Geriatr. Soc. 56, 2234-2243.

Vandenbroucke, A., Luyten, F. P., Flamaing, J., Gielen, E. (2017) Pharmacological treatment of osteoporosis in the oldest old. Clin. Interv. Aging 12, 1065-1077. 